\title{
Integrating flexible electronics for pulsed electric field delivery in a vascularized 3D glioblastoma model
}

\author{
Marie C. Lefevre ${ }^{1}$, Gerwin Dijk ${ }^{1,2}$, Attila Kaszas ${ }^{1}$, Martin Baca ${ }^{1}$, David Moreau ${ }^{1}$ and Rodney P. O'Connor $\mathbb{D}^{1 凶}$
}

Glioblastoma is a highly aggressive brain tumor, very invasive and thus difficult to eradicate with standard oncology therapies. Bioelectric treatments based on pulsed electric fields have proven to be a successful method to treat cancerous tissues. However, they rely on stiff electrodes, which cause acute and chronic injuries, especially in soft tissues like the brain. Here we demonstrate the feasibility of delivering pulsed electric fields with flexible electronics using an in ovo vascularized tumor model. We show with fluorescence widefield and multiphoton microscopy that pulsed electric fields induce vasoconstriction of blood vessels and evoke calcium signals in vascularized glioblastoma spheroids stably expressing a genetically encoded fluorescence reporter. Simulations of the electric field delivery are compared with the measured influence of electric field effects on cell membrane integrity in exposed tumor cells. Our results confirm the feasibility of flexible electronics as a means of delivering intense pulsed electric fields to tumors in an intravital 3D vascularized model of human glioblastoma.

npj Flexible Electronics (2021)5:19; https://doi.org/10.1038/s41528-021-00115-x

\section{INTRODUCTION}

Glioblastoma multiforme (GBM) is a highly aggressive malignant primary brain tumor and one of the most challenging diseases to treat in oncology ${ }^{1}$. Despite available treatments based on surgical resection, radiotherapy, and chemotherapy, the prognosis remains very poor with a median survival time after diagnosis of $\sim 15$ months $^{2}$. Radical resection of the primary tumor mass is not curative due to the high degree of invasiveness of GBM. Infiltrating tumor cells stay within the surrounding brain area, leading to disease progression and recurrence ${ }^{3}$. Even after combining radical resection and multimodal therapies, $\sim 70 \%$ of the cases of GBM patients will experience disease progression within one year of diagnosis ${ }^{4}$. Accordingly, improving existing diagnostics and treatment tools is vital, as well as the development of novel approaches for the treatment of recurrent glioblastoma.

Bioelectric therapies show great potential for the treatment of cancer, although they are poorly clinically applied to treat human brain cancers. Indeed, it still raises skepticism related to a lack of supportive data, especially in GBM treatments ${ }^{5}$. These therapies use intense pulsed electric fields (PEFs) of sufficient intensity to modify the permeability of cellular membranes and influence the tumor microenvironment. One of the most established of these methods is electrochemotherapy (ECT), which improves the intracellular delivery of chemotherapy drugs including bleomycin and cisplatin by the application of $\mathrm{PEFs}^{6}$. The significant advantage of ECT is its selectivity. Indeed, as the threshold for depolarization is higher for excitable cells, it is possible to specifically targets tumor cells without harming neurons, by optimizing pulse parameters, such as pulse duration or electric field intensity ${ }^{6-9}$. Recently, an alternative to ECT has been suggested, based on the enhanced delivery of extracellular calcium ions via electroporation ${ }^{6}$. Calcium ions are involved in many cellular processes and by elevating the intracellular calcium concentration in cells one can enhance tumor necrosis ${ }^{10}$. Irreversible electroporation (IRE) is another well-characterized bioelectric therapy based on PEFs. In this case, electric pulses are used to ablate large volumes of tissue in a non-thermal way by inducing irreversible cell membrane disruption. However, it is still not clear whether cell death occurs by necrosis, apoptosis, or both mechanisms ${ }^{11,12}$. PEFs also disrupt the tumor microenvironment, besides their direct effect on tumors. They trigger neural and nonneural vasoconstriction mechanisms depending on the voltage. Low-voltage stimuli induce reversible vasoconstriction through neural pathways, while high-voltage stimuli activate both pathways, as discussed in more details in Brinton et al. paper ${ }^{13}$. This results in a disruption of the vascular perfusion, which can impact drug delivery and tumor survival ${ }^{14-16}$. All of the above treatments are delivered via the surgical insertion of rigid metal electrodes into tissue for an acute treatment period that lasts in the range of seconds to minutes, followed by removal of electrodes devices.

Insertion of rigid electrode materials into the brain is traumatic to tissue. Implanted metal electrode materials cause several issues, such as mechanical mismatches and foreign body inflammatory responses ${ }^{17}$. The brain is particularly sensitive to insertion and micromotions of implanted stiff electrodes, which are known to causes acute and chronic injuries due to inflammation and astroglial scarring ${ }^{18}$. In addition to being invasive and mechanically non-conformable, currently used electrodes mainly target small areas and demand the application of high voltage pulses, which can cause damage to nearby healthy tissue the brain ${ }^{19}$.

The emergence of plastic bioelectronic technologies opens the way for less invasive implanted treatments. Compared to metal or silicon-based electrodes that are rigid, thin conductive plastic film electrodes have lower Young's modulus ${ }^{20}$. Their use as substrates results in more flexible, soft electrodes that have better compliance with biological tissues. In addition to their good mechanical properties, plastic electronics allows large-area sensing and stimulation on curvilinear surfaces ${ }^{21}$. We propose to integrate flexible electronic technology into tumors for the treatment of cancer with implanted devices using their delivered electric fields.

In testing electrode technologies, one typically starts with in vitro models before in vivo preclinical experiments on animals.

${ }^{1}$ Mines Saint-Etienne, Centre CMP, Department of Bioelectronics, Gardanne, France. ${ }^{2}$ Panaxium SAS, Aix-en-Provence, France. ${ }^{凶}$ email: rodney.oconnor@emse.fr 


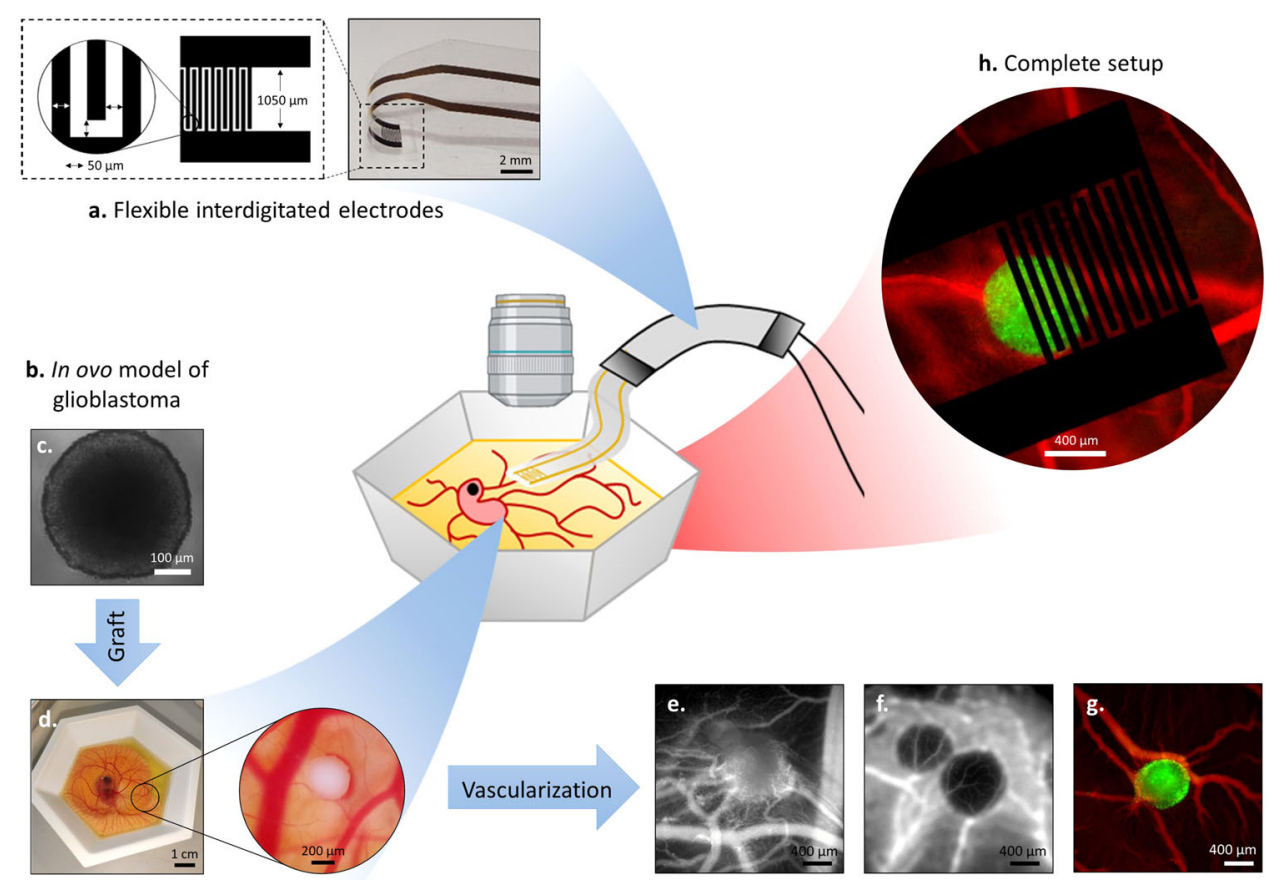

Fig. 1 Integration of flexible electronics onto a vascularized 3D tumor model. a Flexible interdigitated electrodes and the scheme of the tip. b Steps of the development of the in ovo model of glioblastoma. c Spheroid of U87 cells obtained by 3D cell culture observed under the microscope. d Shell-less culture of a quail embryo with a zoom on the grafted spheroid. e-g pictures of vascularized spheroids observed by fluorescence microscopy. On e and $\mathbf{f}$, vasculature was visualized by injecting Texas Red dextran into the circulation. On (g) in addition to the intravascular injection of Texas Red dextran in the circulatory system (red), spheroid was labeled intravitally with a viability marker (green). h Flexible interdigitated electrodes placed on the in ovo model of glioblastoma, observed by fluorescence microscopy with the same markers than (g). Scale bars: a $2 \mathrm{~mm}$, c $100 \mu \mathrm{m}$, d left: $1 \mathrm{~cm}$, right: $200 \mu \mathrm{m}$, e-h $400 \mu \mathrm{m}$.

Although cost-effective, convenient, and easy to set up, in vitro models have many limitations as they fail to mimic the complex microenvironment of native tumors ${ }^{22}$. In vivo models are more robust but also more expensive, time-consuming, and raise ethical issues. For this reason, intermediate models are an interesting alternative for testing and developing therapies. The in ovo model presented in this work combines a living organism-a quail embryo-and an in vitro 3D model of glioblastoma tumor. Indeed, three-dimensional cell culture allows one to generate spheroids of glioblastoma cells. These spheroids exhibit many features of avascular tumors, such as gradients in oxygen and nutrients, or a necrotic core ${ }^{23-25}$. Engineered tumors are grafted into the embryonic membrane of a quail, the chorioallantoic membrane (CAM). This membrane is highly vascularized and has been extensively used to study angiogenesis and the efficiency of the drug delivery to tumor models ${ }^{26,27}$. At an early stage of development, quail embryos have no immune system, thus tumors can be grafted in the CAM without being recognized as a foreign body ${ }^{26}$. Spheroids of glioblastoma secrete growth factors, mainly because of hypoxia, which induces angiogenesis ${ }^{28-30}$. As a consequence, new blood vessels will grow from the existing vasculature of the quail embryo, vascularizing the tumor and supplying nutrients and oxygen ${ }^{31}$.

In this work, we present a complete model for the study of a flexible electronic PEF delivery system implanted onto glioblastoma tumors. We first describe the development of the 3D vascularized biological model of glioblastoma and then use this model to deliver PEFs with flexible electronics to spheroids of GCaMP6f-expressing glioblastoma cells grafted in the chorioallantoic membrane. Next, we demonstrate the influence of PEFs delivered by our flexible interdigitated electrodes on the vascular constriction of blood vessels. Finally, we investigate the dosedependency of PEF-induced calcium signals in vascularized spheroids, measuring the influence of repeated applications of
PEFs on tumor cell membrane integrity with widefield fluorescence and two-photon microscopy.

\section{RESULTS}

\section{D model of glioblastoma and integration of flexible electronics}

A vascularized human brain cancer model was used as a platform for testing flexible electronics as a means of delivering pulsed electric fields to tumors. Interdigitated electrodes were designed to deliver an electric field between electrode fingers (Fig. 1a) ${ }^{19}$. Gold electrodes were deposited onto a thin layer of the organic polymer Parylene-c, resulting in a flexible and biocompatible device $20,32,33$. This substrate is transparent, allowing fluorescence imaging through the device.

For the development of the in ovo model of glioblastoma, several techniques were combined (Fig. 1b). The liquid-overlay 96well plate method of 3D-cell culture was used to produce spheroids of glioblastoma mimicking avascular brain tumors (Fig. 1b.1). In parallel, quail eggs were opened to obtain a shellless culture of quail embryos (Fig. 1b.2). The graft of the spheroids in the CAM induced vascularization of the tumor (Fig. 1b.3-5). The flexible electronic device was placed directly on the vascularized tumor and the effect of PEFs was measured with fluorescence microscopy (Fig. 1c).

The flexibility and the biocompatibily of our electrodes were key points to have a good interaction with tissues, first with the in ovo model of GBM, but also with brain in the future. The substrate chosen for this purpose was the Parylene-c. This organic polymer has a much lower Young modulus $(2.76 \mathrm{GPa})$ than standard stiff silicon electrodes (150-170 GPa), which reduce significantly the mechanical mismatch with soft brain tissues $(3-100 \mathrm{kPa})^{34}$. The use of this polymer resulted in a highly conformable probe, which adjusts to curvilinear surfaces as demonstrated by placing it on 

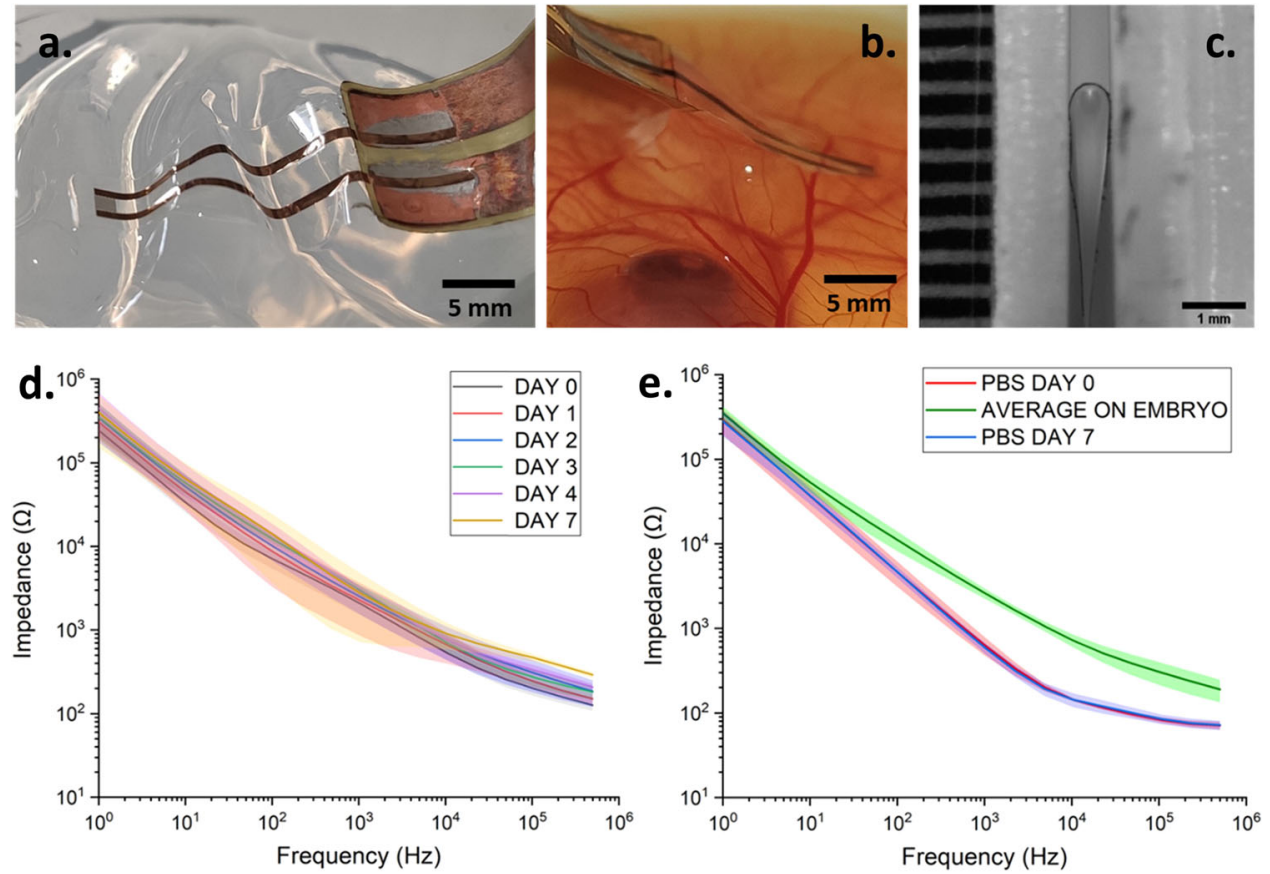

Fig. 2 Mechanical and electrical characterization of flexible electrodes. a Flexible device placed on the surface of a phantom brain made in $0.6 \%(\mathrm{w} / \mathrm{v})$ agarose in deionized water. b Flexible device sticking to the CAM. c Flexible device bended between two jaws, with a bending radius of $0.2 \mathrm{~mm}$. d Electrochemical impedance spectroscopy of the flexible interdigitated electrodes in situ over time. DAY 0 to 2: $N=5$, DAY 3 and 4: $N=4$, DAY 7: $N=2$ ). e Impedance spectroscopy in vitro in PBS, before and after the experiment, and the average impedance in situ. $N=5$. Error bars indicate standard deviations. Scale bars: a, b $5 \mathrm{~mm}$, c $1 \mathrm{~mm}$.
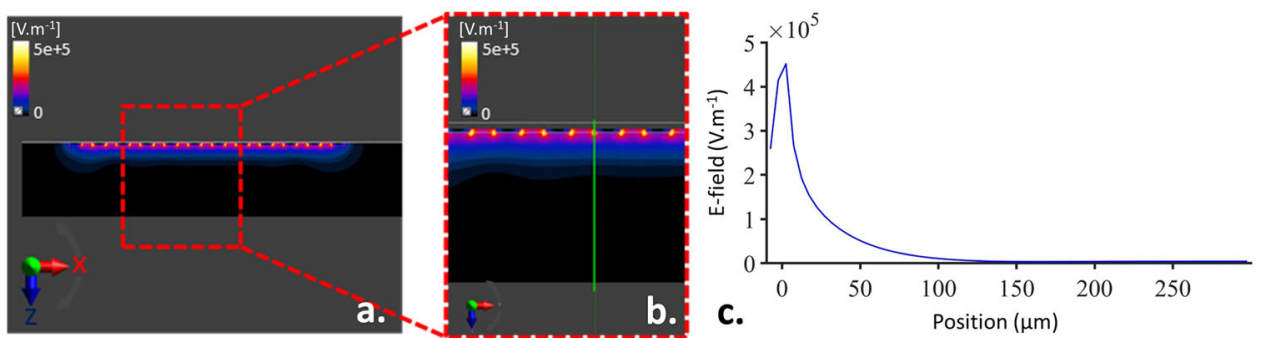

Fig. 3 Numerical simulations of the electric field. a Spatial distribution of the electric field as a function of distance from the electrodes. $\mathbf{b}$ Zoom on a region of interest with only subset of interdigitated electrodes. c View of the electric field magnitude along the green line represented in b.

the irregular surface of a phantom brain made in agarose (Fig. 2a). The surface of the device is hydrophilic, as the measured static contact angle with water is $68.8 \pm 5.2^{\circ}(n=3)^{35}$, and it sticks to the membrane of the embryo (Fig. $2 \mathrm{~b}$ and Supplementary Video 1). Finally, it has a good resistance to bending as it was still functional after reaching a bending radius of $0.2 \mathrm{~mm}$ (Fig. 2c).

The biocompatibility of the flexible electrodes was assessed by performing electrochemical impedance spectroscopy (EIS). Different probes $(n=5)$, either new or used for previous experiments, were placed in situ on the chorioallantoic membrane of the embryos $(N=5)$ on DAY 0 and the EIS was measured every day (Fig. 2d). From DAY 0 to 7, no significant changes were observed in the electrode impedances (Fig. 2e). The stable impedance values confirm that both the electrode integrity is intact and that there were no significant changes in biological environment, and so no foreign body response, which was previously reported to increase electrode impedance over time ${ }^{36}$. Moreover, no visual damage was observed on the embryo after removal of the probe, in contrast with the bleeding caused by the insertion of standard stiff electrodes (Supplementary Fig. 1). Figure 2e shows the impedance measured in vitro in phosphate-buffered saline (PBS) before and after the experiment, as well as the average impedance on the embryo. The impedance in situ was higher than in vitro for frequencies $>100 \mathrm{~Hz}$ which is typical for an epithelial cell layer that covers an electrode ${ }^{37}$. No change was observed in the impedance before and after the experiment, indicating no electrode degradation and stable performance in time.

In order to understand the dosimetry of the flexible device, numerical simulations were performed to model the spatial distribution of the electric field delivered to the biological model. The distribution of electric field was investigated at the electrode in contact with a saline solution on a $300 \mu$ m-thick layer. Using the electro-ohmic quasi-static solver (Sim4life, Zurich), the spatial distribution was simulated evaluating the intensity of the electric field as a function of the distance from the electrodes in the $X Z$ plane (Fig. 3a, b). Results showed that the electric field decreased to less than $1 \%$ of the maximum intensity at a distance of $130 \mu \mathrm{m}$ from the surface of the microelectrodes (Fig. $3 \mathrm{c}$ ), demonstrating the highly focal surface delivery of our flexible electronic device.

U87-spheroids were formed using a variation of the liquidoverlay 96-well plate method. The target was obtaining spheroids 

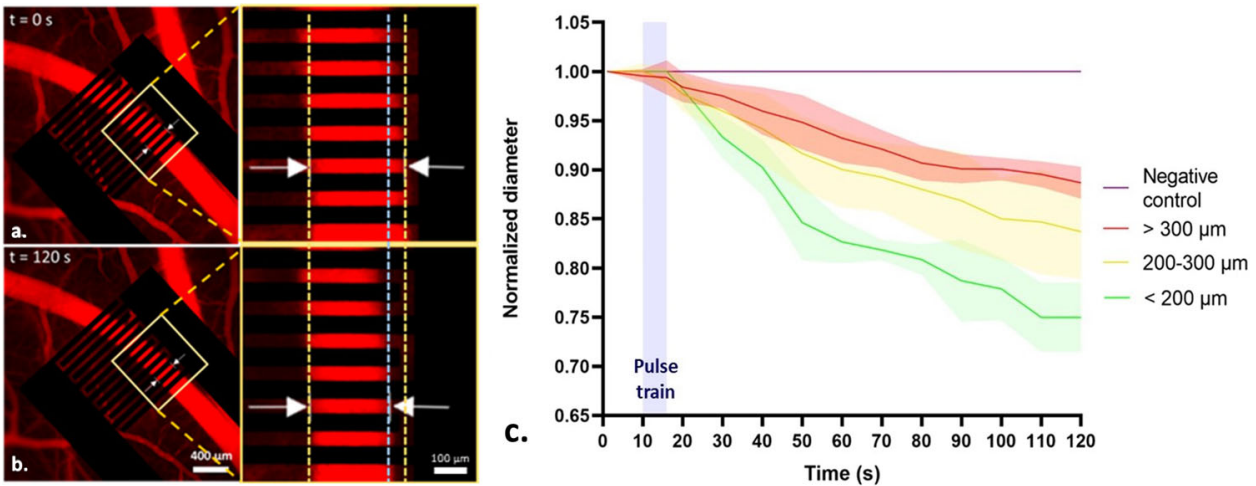

Fig. 4 Vasoconstriction induced by flexible electronic delivery of PEFs. a, b view from the top of the device placed on the vascularized tumor and a zoom on the region of interest at the beginning (a) and the end (b) of the experiment. Texas Red dextran was injected into the vascular circulation to visualize blood vessels with fluorescence microscopy at $\lambda_{\text {exc }}=595 \mathrm{~nm}$. White arrows show the location of the measure, performed between the dotted lines depicting the edges of the blood vessel at the beginning (yellow) and the end (blue) of the experiment. Scale bars: (a, b) left panel: $400 \mu \mathrm{m}$, right panel: $100 \mu \mathrm{m}$. b Normalized diameter over time after PEFs delivery $(5$ pulses-20 V-100 $\mu \mathrm{s}-1 \mathrm{~Hz})$ depending on the blood vessel diameter. The pulse train is represented in blue. Negative control: $N=3$, large blood vessels: $N=6$, medium blood vessels: $N=11$, small blood vessels: $N=3$. Error bars indicate standard deviations.

with a diameter exceeding $400 \mu \mathrm{m}$ to induce the formation of a hypoxic core ${ }^{38}$. Indeed, without supply of oxygen and nutrients provided by the blood, avascular tumors may become necrotic or apoptotic $^{39}$, which in turn triggers the angiogenic switch, a transition between the avascular state and a stage of active blood vessel growth mainly driven by hypoxia ${ }^{28}$. Hypoxic cells in the tumor express growth factors such as vascular endothelial growth factor (VEGF), which induce angiogenesis and thus the formation of new blood vessels that can supply the tumor ${ }^{28-30}$.

Next, the goal was achieving spherical spheroids, meaning a coefficient of roundness above 0.8 (see Materials and methods for calculations), in order to be reproducible between experiments. The roundness and diameter of the spheroids were studied, depending on the number of cells seeded and the time. After 7 days of growth, the majority of spheroids were spherical with a diameter above $400 \mu \mathrm{m}$, except for spheroids made of 5000 cells, whose diameter was $387 \pm 17 \mu \mathrm{m}$ ( $n=44$ spheroids). A total of 10,000 cell-spheroids, 15,000 cell-spheroids, and 20,000 cellspheroids had respective diameters of $443 \pm 11 \mu \mathrm{m}, 474 \pm 13 \mu \mathrm{m}$, and $487 \pm 14 \mu \mathrm{m}$ ( $n=44$ spheroids). In total 10,000 cell-spheroids were arbitrary chosen for the rest of the experiments.

After implantation of a tumor in the CAM, embryos were observed with fluorescence microscopy. Neo-angiogenesis was observed within 1-2 days as blood vessels developed from the CAM to irrigate the implanted tumors. Some grafts were performed using the viability marker Calcein-AM to label spheroids (Fig. 1b5) to illustrate the extent of this vascularization.

\section{Flexible electronic delivery of PEFs to the vasculature of the embryo}

PEFs have a known effect on the tumor microenvironment as they disrupt perfusion by blood vessels, and thus lead to reduced blood flow and oxygenation in tumors. The study of this phenomenon is important for bioelectric therapies such as ECT, as it can affect the kinetics of drug delivery ${ }^{14,40}$. We first used our model without grafting a tumor to validate the delivery of electric fields by our flexible electronic device.

Experiments were performed on a random selection of blood vessels. A pulse train of $5,100 \mu \mathrm{s}$ monophasic voltage pulses were delivered at $20 \mathrm{~V}$ with a resulting current measured at the terminals of $100 \mathrm{~mA}$. After applying PEFs, the blood vessels near the microelectrodes showed vasoconstriction (Supplementary Video 2). One example shown is that of a $296 \mu \mathrm{m}$-wide blood vessel (Fig. 4a, b). After $120 \mathrm{~s}$ of beginning of the experiment, the diameter decreases to $257 \mu \mathrm{m}$, corresponding to a vasoconstriction of $13 \%$.

Normalized diameters of blood vessels were compared over time to evaluate flexible electronic delivery of PEFs (Fig. 4c). Blood vessels were grouped depending on their diameter: large (more than $300 \mu \mathrm{m}$ ), medium (between 200 and $300 \mu \mathrm{m}$ ) and small (less than $200 \mu \mathrm{m}$ ). With the same parameters for the delivery of PEFs, diameters decreased of $11.3 \pm 1.6 \%$ (Mean $\pm S D$ ) for large blood vessels ( $N=6$ blood vessels from $n=4$ eggs), $16.3 \pm 4.8 \%$ for medium blood vessels ( $N=11$ blood vessels from $n=7$ eggs) and $25 \pm 3.5 \%$ for small blood vessels $(N=3$ blood vessels from $n=2$ eggs). Negative controls were performed without pulse delivery showing vessel diameter remained stable throughout all experiment, indicating that vasoconstriction was indeed due to PEF delivery.

\section{Flexible electronic control of tumor calcium signals}

PEFs delivery to tumors by flexible electrodes was confirmed by measuring calcium signals by fluorescence imaging in vascularized spheroids. The flexible interdigitated device was implanted onto the vascularized tumor preparation and PEFs were delivered at a series of voltages to determine the dose-response relationship (Fig. 5a). Fluorescence intensity was normalized to initial signals and measured over time thereafter. The change in fluorescence, and thus tumor cell intracellular calcium, was shown to increase as a function of the applied voltage as shown in Fig. 5b. A significant increase in fluorescence was observed immediately after pulse delivery with an EC50 of $16.3 \mathrm{~V}$, where the EC50 is the voltage at which a half-maximum response is reached. Control experiments, where no voltage pulse was delivered showed no change in fluorescence.

\section{Manipulation of tumor cell membrane integrity by flexible electronic delivered PEFs}

Flexible electrodes implanted onto the biological preparation were used to deliver repeated application of PEFs to disrupt the membrane integrity of tumor cells. We compared the uptake of the red fluorescent indicator propidium iodide (PI) into vascularized tumor spheroids following repeated pulse trains. PI is a nucleic acid-binding fluorophore that is cell impermeant and thus, can enter only in cells with compromised membranes ${ }^{41}$. It is commonly used as an indicator of electropermeabilization following the delivery of pulsed electric fields. Trains of 5 pulses $(20 \mathrm{~V}-100 \mu \mathrm{s}-1 \mathrm{~Hz}$ ) were repeated every $30 \mathrm{~s}$ and the fluorescence 

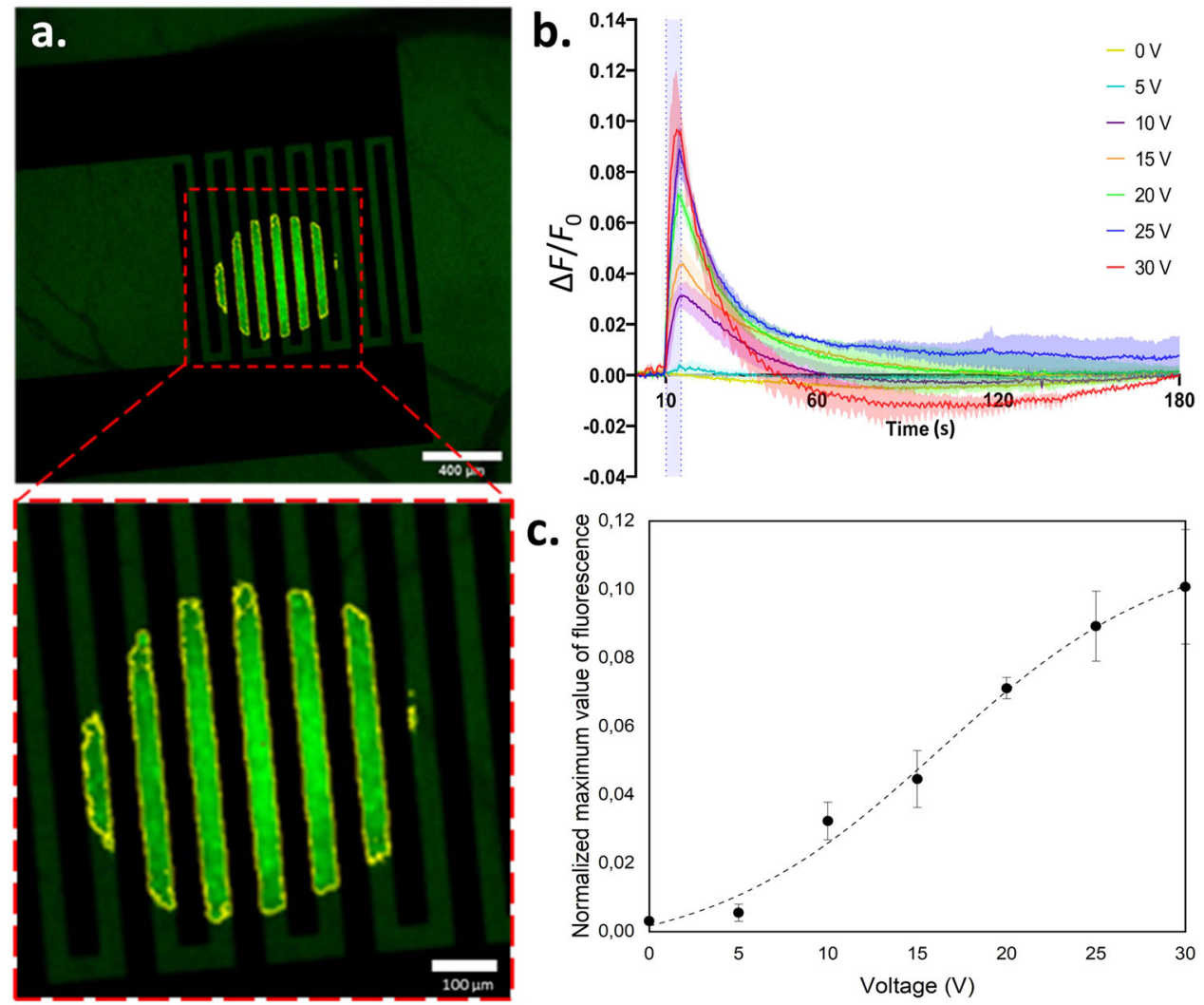

Fig. 5 Effect of PEFs on calcium signaling. a Picture from the top of the device placed on a U87-GCaMP6f spheroid grafted in the CAM observed at $\lambda_{\text {exc }}=490 \mathrm{~nm}$ and a zoom on the region of interest. The fluorescence is measured on the ROI depicted in yellow. Scale bars are respectively $400 \mu \mathrm{m}$ and $100 \mu \mathrm{m}$. b Normalized fluorescence intensity measured over time depending on the voltage applied. c Dose-response: maximum fluorescence change depending on the voltage applied: $0 \mathrm{~V}(n=7), 5 \mathrm{~V}(n=5), 10 \mathrm{~V}(n=4), 15 \mathrm{~V}(n=5)$, $20 \mathrm{~V}(n=3), 25 \mathrm{~V}(n=3), 30 \mathrm{~V}(n=2)$. Error bars indicate standard deviations.

of the spheroids was compared (Fig. 6a). An increase in PI fluorescence was observed after applying 4, 10, or 20 trains of PEFs, compared to control experiments, which was performed without delivering PEFs resulting in no change in fluorescence. In parallel, we followed the change in GCaMP6f, representing tumor intracellular calcium over time to assess the influence of repeated delivery of PEFs (Fig. 6b). An increase of fluorescence was observed following each train of pulses, but the amplitude was shown to decrease over time.

The depth of the electric field influence on tumor cell membrane integrity was verified by two-photon microscopy, which showed that spheroids pulsed with flexible electrodes (Fig. 6d) showed increased PI fluorescence, as compared to control samples (Fig. 6c). Analysis of the cross-section of the spheroid before and after pulsing (trains of 5 pulses $(20 \mathrm{~V}-100 \mu \mathrm{s}-1 \mathrm{~Hz})$ repeated 10 times), confirmed that the increase in PI fluorescence (Fig. 6f), and thus electropermeabilization, occurred at a depth (Fig. 6g) similar to that predicted by our model (Fig. 3.c). The obtained results show that repeated stimulation by flexible electrodes induces $\mathrm{PI}$ uptake in vascularized tumor spheroids, demonstrating electropermeabilization at the surface of the tumor, as predicated by simulations of the electric field under the device surface.

\section{DISCUSSION}

In this work, we present a complete model permitting the integration of flexible electronics into intravital vascularized glioblastoma tumors. The combination of an optimized liquidoverlay 96-well plate method and the ex ovo CAM model allowed us to produce vascularized brain tumors. This preparation is a good compromise between in vitro and in vivo experiments, as it is a more complex model of solid tumor compared to cell culture monolayers, without the inconvenience, cost, and ethical issues associated with animal models. Experiments with this preparation are only limited in time by the survival of the embryo up to 12 days, thus allowing 7 days of observation. The method is inexpensive, fast, reproducible, high-throughput, and raises less ethical issues (requiring no animal experimentation authorization in Europe). It allows complete access to the circulatory system of the embryo, which can be useful for following drug uptake. As the embryo has no immune response, there is no foreign body response either to the xenograft tumor (human) or the electronics, as demonstrated by impedance spectroscopy. It is therefore an outstanding system for testing flexible electronic devices under intravital conditions before moving towards preclinical animal studies.

We used our model to investigate vasoconstriction triggered by PEFs to demonstrate that flexible electronics are capable of bioelectric therapies previously shown in our work with rigid metal electrodes ${ }^{15}$ and others ${ }^{42}$. Vasoconstriction of blood vessels exposed to PEFs was observed to depend on their diameter, as previously shown ${ }^{42}$. This decrease of diameter implies an even greater decrease of the blood flow as the flow rate in a cylindrical pipe is proportional to the fourth power of the diameter (Poiseuille's equation) ${ }^{43}$.

Some variability was expected as the electric field decreases with the distance from the electrodes in the orthogonal direction ${ }^{44}$. We showed that this model is suitable for the study of vascular effects of PEFs, which may be used to develop implantable flexible electronic devices to reversibly control blood perfusion for applications in surgery or wound healing. 


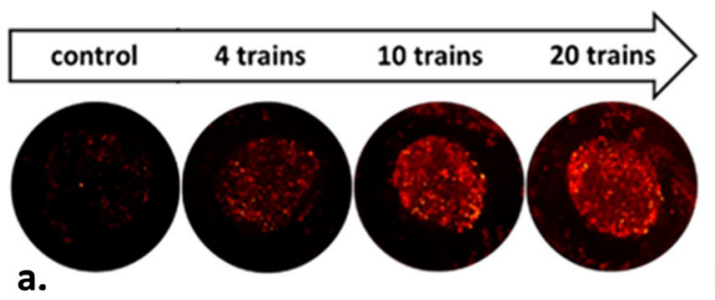

a.
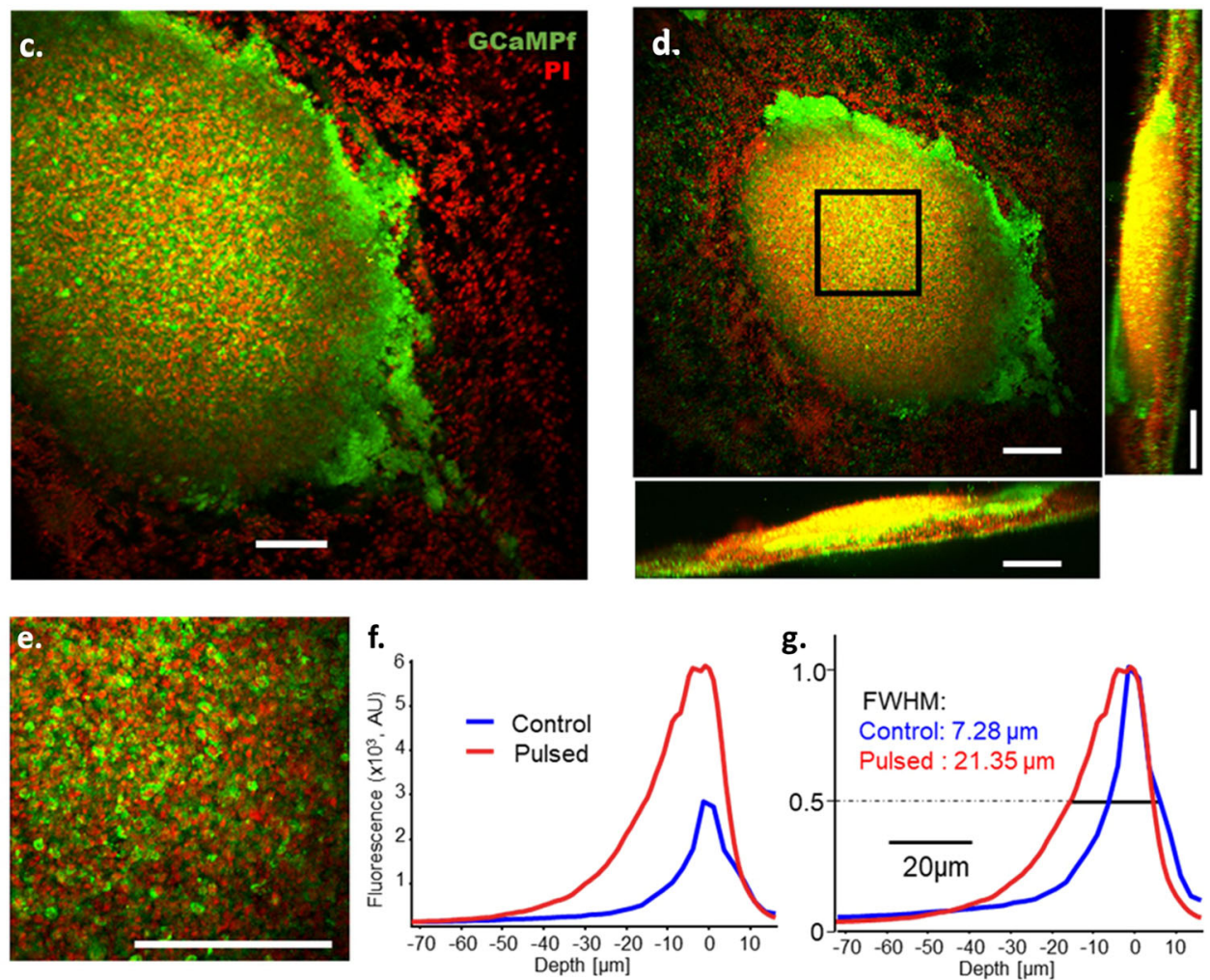

Fig. 6 Uptake of PI due to PEFs. a Spheroids observed at $\lambda_{\text {exc }}=550 \mathrm{~nm}$ before and after application of PEFs. b Normalized fluorescence intensity of calcium signals $\left(\lambda_{\text {exc }}=490 \mathrm{~nm}\right.$ ) measured over time of spheroids subjected to 10 trains of PEFs (red ticks). Trains of 5 pulses $(20 \mathrm{~V}-100 \mu \mathrm{s}-1 \mathrm{~Hz})$ are delivered every $30 \mathrm{~s}$. c, d Two-photon maximum intensity projections of implanted spheroids at (c) control and (d) pulsed states (five $20 \mathrm{~V}$ pulses with $100 \mu \mathrm{s}$ pulse width at $1 \mathrm{~Hz}$, repeated 10 times at $30 \mathrm{~s}$ interstimulus intervals). e Zoom-in to the region marked with the square on $\mathbf{d}$. $\mathbf{f}-\mathbf{g}$ Raw $(\mathbf{f})$ and normalized $(\mathbf{g})$ side projection pixel intensity profile of propidium-iodide staining from control spheroid (blue) and after pulsing (red). FWHM: full width at half maximum. Scale bars: $100 \mu \mathrm{m}$.

Finally, we demonstrated the potential of flexible electronics to control cellular signals in vascularized tumors stably expressing a fluorescent calcium sensor. Stimulation via the device was capable of elevating intracellular tumor calcium in a dosedependent manner with the applied electric field. Significantly, transient and reversible increases in GCaMP6f fluorescence were measured at applied voltages ranging from $10 \mathrm{~V}$ to $30 \mathrm{~V}$, showing it was possible to repeatably stimulate intracellular calcium signals in tumor cells.

Two-photon microscopy was used to show the focal nature of the electric field delivered by the flexible electrode device as a function of tissue depth in tumors, confirming numerical simulations. As expected, cells in the first $20-25 \mu \mathrm{m}$ of the interdigitated electrode surface were electropermeabilized. Repeated electrical stimulations and subsequent increases in GCaMP6f fluorescence intensity in glioblastoma spheroids, showed that calcium signals in these tumors could be controlled with the device. The intracellular calcium concentration of cells is tightly regulated in cells and serves as a universal signal ${ }^{45}$. Prolonged excursions of intracellular calcium can be used to induce apopotosis or necrosis, and indeed is the means by which calcium electroporation can be used to kill cancer by the combination of electropermeabilization in the presence of high extracellular concentrations ${ }^{10}$. Adding extracellular calcium has some risks in the brain as it can influence neuronal excitability by interactions with membrane phospholipids that alter the intramembrane electric field or through Calcium sensing receptors ${ }^{46,47}$.

Here we propose a means of dysregulating intracellular calcium in tumor cells by repeated stimulation and electrical control with an implanted flexible electronic device. Although the custom pulse generator used in the current study was not designed to be left in the biological preparation, an implantable stimulator system for integration with the flexible electrode system is under development for close loop stimulation.

Flexible and biocompatible interdigitated microelectrodes were designed and validated in this work for their capability to deliver PEFs and disrupt the tumor microenvironment. These electrodes are highly conformable and can be implanted on the surface of the CAM or tumor without affecting viability of the embryo. Moreover, they remain stable in biological environments and can be reused. The use of a transparent substrate allowed optical imaging with widefield fluorescence and multiphoton microscopy through the device. The design can easily be adapted as standard techniques of flexible electronic microfabrication are used. Future 
microfabrication strategies can include the addition of thin layers of the conductive polymers PEDOT:PSS to increase charge injection to the biological sample ${ }^{48}$, or implementation of organic ion pumps to electronically elute charged chemotherapeutics via iontophoresis 49,50 .

\section{METHODS}

\section{Tumor cell lines and culture}

Human U87-MG glioblastoma cells (ATCC HTB-14) were purchased from Sigma and maintained at $37{ }^{\circ} \mathrm{C}$ in a $5 \% \mathrm{CO}_{2}$ humidified atmosphere. Cells were cultured in Dulbecco's Modified Eagle's Medium + GlutaMAX ${ }^{\mathrm{TM}}$-I (10567-014, Gibco) supplemented with 10\% fetal bovine serum (S181BH, VWR), 100 units $\mathrm{mL}^{-1}$ of penicillin, and $100 \mathrm{\mu g} \mathrm{ml}^{-1}$ of streptomycin (15140-122, Gibco)

\section{Generation of U87-GCaMP6f stable cell line}

U87 cells were genetically modified by lentiviral transduction. Lenti-X $293 \mathrm{~T}$ cell line (63218, Takara Bio) is a HEK 293T-derived cell line optimized for Lenti-X virus production, which is able to deliver a gene of interest into cells. Lenti-X 293 T cells were cultured in Dulbecco's Modified Eagle's Medium containing $4.5 \mathrm{~g} \mathrm{~L}^{-1}$ of glucose, L-glutamine, sodium pyruvate, and sodium bicarbonate (D6429, Sigma), supplemented with $10 \%$ tetracycline-free FBS (631105, Takara Bio), 100 units $\mathrm{mL}^{-1}$ of penicillin and $100 \mu \mathrm{g} \mathrm{ml}^{-1}$ of streptomycin (15140-122, Gibco). pGP-CMV-GCaMP6f (40755, Addgene) is a plasmid created by Douglas Kim containing the gene GCaMP6f and a selection marker conferring resistance to puromycine $^{51}$. Plasmid DNA was extracted with GeneJET plasmid Maxiprep Kit (K0492, Thermofisher) following the protocol given by the supplier.

For the production of the virus containing the gene of interest, Xfect single shot transfection reagent (631447, Takara) was used following the protocol provided by Takara (111814). Briefly, $25 \mu \mathrm{g}$ of plasmid DNA was diluted in sterile water for a final volume of $600 \mu \mathrm{L}$ and added to a tube of Xfect single shots reagent. After 10 seconds of vortexing, the tube was incubated $10 \mathrm{~min}$ at room temperature to allow the production of nanoparticles. The content of the tube was added dropwise on the culture of Lenti-X $293 \mathrm{~T}$ cells. Cells were incubated at $37^{\circ} \mathrm{C}$ for at least $4 \mathrm{~h}$. Then, the virus production in the supernatant was confirmed by using Lenti- $X^{\text {TM }}$ GoStix ${ }^{\text {TM }}$ Plus (631280, Takara).

Before the infection with the lentivirus, U87-MG cells were grown for 4 days in Dulbecco's Modified Eagle's Medium + GlutaMAX ${ }^{\mathrm{TM}}-\mathrm{I}$ (10567-014, Gibco) supplemented with $10 \%$ tetracycline-free FBS (S181BH, VWR), 100 units $\mathrm{mL}^{-1}$ of penicillin and $100 \mu \mathrm{g} \mathrm{ml}^{-1}$ of streptomycin (15140-122, Gibco). Supernatant containing the virus was added on the target cells with $5 \mu \mathrm{g} \mathrm{mL}^{-1}$ of Sequa-brene (S2667, Sigma), a product that enhances the efficiency of the lentiviral infection. After $6 \mathrm{~h}$ of incubation at $37^{\circ} \mathrm{C}$, the medium was replaced by a fresh one. Transducted cells were selected by using $0.5 \mu \mathrm{g} \mathrm{mL}^{-1}$ of puromycine (A11103, Gibco) in the media. A stable U87-GCamp6f cell line was generated from a single transducted cell.

\section{Tumor spheroids}

Spheroids were produced using a variation of the liquid-overlay 96-well plate method ${ }^{52}$. A solution of $1 \%(\mathrm{v} / \mathrm{w})$ agarose (Sigma Aldrich) was prepared in deionized water and autoclaved. After heating up the solution one minute in the microwave, $75 \mu \mathrm{L}$ was added in each well to form a meniscus on the bottom of the well. After 10 minutes at room temperature, the agarose cooled down and jellified to form a nonadherent round-bottom well. Glioblastoma cells were rinsed with Phosphate Buffer Saline solution (Thermofisher D8537) and detached using $1 \mathrm{~mL}$ of $0.25 \%$ Trypsin-EDTA (1X) (Gibco 25200-056) at $37^{\circ} \mathrm{C}$. The desired quantity of cells was added per well, in a total volume of $150 \mu \mathrm{L}$ per well. Half of the media was changed every 2-3 days. This method of generating spheroids is reproducible, inexpensive and high-throughput. Indeed, it requires 7 days and only very little material to produce hundreds of spheroids with a similar size exceeding $400 \mu \mathrm{m}$. Moreover, spheroids are easy to handle and can be collected and grafted using a standard $1000-\mu \mathrm{L}$ pipette.

\section{Growth monitoring of spheroids}

To study the growth of spheroids over time, $2 \times 10^{4}$ cells, $1.5 \times 10^{4}$ cells, $1 \times 10^{4}$ cells and $5 \times 10^{3}$ cells in $150 \mu \mathrm{L}$ of medium were respectively added in 24 wells of a 96 -well plate. Half of the medium was changed every
2-3 days. Spheroids were analyzed from day 6 to day 15 using the software Zen 2 Lite Carl Zeiss Microscopy GmbH with a microscope and a camera from Carl Zeiss. Roundness is determined with the software ImageJ, by fitting an ellipse to the spheroid. The square of the length of its primary axis is compared to the square of the diameter of a circle of the same area to determine the roundness. The calculation is based on Eq. (1):

Roundness $=4 \times \frac{\text { Area }}{\pi \times[\text { Major Axis }]^{2}}$

Area and [Major Axis] correspond respectively to the area and to the length of the primary axis of the best fitting ellipse. A roundness of 1 corresponds to a circle whether a roundness of 0 corresponds to an elongated ellipse.

The measure of the diameter was performed with the software Zen 2 Lite, only on spheroids with a coefficient of roundness over 0.8 . The diameter of each spherical spheroid was measured on a view from the bottom.

\section{Ex ovo CAM assay}

The chorioallantoic membrane (CAM) assay was developed with quail eggs and adapted from previously described protocols ${ }^{15,31}$. Fertilized eggs of Japanese quail (coturnix japonica) were obtained from Japocaille (Saint Euphrône, France) and incubated at $37^{\circ} \mathrm{C}$ on trays with an automatic rotator that turned eggs every $2 \mathrm{~h}$, with a humidity of $57 \%$. The day when eggs were set in the incubator was considered as the Embryonic Day (ED) 0 . On ED3, the eggs were gently opened and the embryos were poured into a plastic weigh boat, covered with a second weigh boat, and transferred to a standard humidified incubator at $37^{\circ} \mathrm{C}$ as shell-less culture. On ED6, a small incision was performed with a needle to open the CAM membrane. A spheroid was placed on the incision with a pipette and the embryo was returned to the incubator until further experiments.

To assess the viability of the tumor after grafting, spheroids of U87-MG were incubated for $30 \mathrm{~min}$ at $37^{\circ} \mathrm{C}$ with $5 \mu \mathrm{M}$ Calcein-AM (BD Biosciences 354216) solution, a viability marker, before the graft. Except for this experiment, spheroids of U87-GCamp6f were used.

\section{Fabrication of flexible and biocompatible microelectronics}

Interdigitated electrodes were made in our cleanroom. Each electrode had 6 interdigitated fingers of $50 \mu \mathrm{m}$ with an interelectrode gap of $50 \mu \mathrm{m}$. The fabrication process was based on methods reported previously ${ }^{53}$. In total of $7 \mu \mathrm{m}$ of parylene $\mathrm{C}$ were deposited by a SCS Labcoater 2 on a clean glass slide. Metal electrodes and connection leads were patterned using a lift-off process with a bi-layer of LOR5A resist and S1813 photoresist. Photoresist was exposed with a SUSS MBJ4 contact aligner. An adhesion layer of $10 \mathrm{~nm}$ chromium and $150 \mathrm{~nm}$ of gold were evaporated with a Boc Edwards thermal evaporator and the photoresist was lifted off. The outline of the probe was etched with a scissor. Finally, the device was washed with deionized water to delaminate the device from the glass slide.

\section{Characterization of flexible microelectronics}

The conformability of the device was tested on a phantom brain made in $0.6 \%(\mathrm{w} / \mathrm{v})$ agarose in deionized water. The maximum bending radius was measured with a lab-made machine. The static contact angle with water was measured 3 times with a goniometer (Apollo Instrument OCA200).

Electrochemical impedance spectroscopy was performed with a PalmSens 4 from $1 \mathrm{~Hz}$ to $500 \mathrm{kHz}$ and 3 measurements points per decade. A 2-electrode setup was used where one of the interdigitated electrodes functioned as the working electrode and the other as the counter electrode. Measurements were performed each day, e.g., every $24 \mathrm{~h}$.

\section{Numerical modeling}

Simulations of the electromagnetic dosimetry were performed using Sim4Life developed by ZMT, with the Electro Ohmic Quasi-static solver. In order to characterize the device, calculations have been done for $20 \mathrm{~V}$ on a saline solution. The parameters used for the simulations are described in Table 1.

\section{PEF delivery on the CAM model}

PEFs were delivered using a pulse generator built in our laboratory. In total 5 positive pulses of $100 \mu \mathrm{s}$ with a repetition frequency of $1 \mathrm{~Hz}$ were delivered. The voltage was set with a precision of $1 \mathrm{~V}$ at the desired value. The current and the voltage were measured at the terminals of the 
Table 1. Parameters used for the simulations.

\begin{tabular}{|c|c|c|c|}
\hline & $\begin{array}{l}\text { Relative } \\
\text { permittivity } \\
\varepsilon_{\mathrm{r}}\end{array}$ & $\begin{array}{l}\text { Electrical } \\
\text { conductivity } \\
\sigma\left(\mathrm{S} \mathrm{m}^{-1}\right)\end{array}$ & $\begin{array}{l}\text { Thickness } \\
(\mu \mathrm{m})\end{array}$ \\
\hline Gold & 1 & $4.098 \times 10^{7}$ & 0.1 \\
\hline Parylene-c & 3.1 & $1 \times 10^{-13}$ & 7 \\
\hline Saline solution & 77 & 1.5 & 300 \\
\hline
\end{tabular}

electrodes using National Instruments system. Each assay lasted $180 \mathrm{~s}$ and the train of pulses started after $10 \mathrm{~s}$ of the experiment.

For the experiments on vasoconstriction, flexible electrodes were placed directly on a random selection of blood vessels with a diameter in the range of 70 to $483 \mu \mathrm{m}$ wide ( $N=23$ blood vessels in $n=11$ embryos). The electrodes were placed directly under the microscope thanks to an electrophysiology micromanipulator (Sutter Instruments, MPC-200) implemented with a lab-made holding device on which the probe is fixed. The voltage was set to $20 \mathrm{~V}$, corresponding to $4 \mathrm{kV} \mathrm{cm}^{-1}$ for a gap of $50 \mu \mathrm{m}$ between the fingers. Dextran Texas Red $70,000\left(10 \mathrm{mg} \mathrm{mL}^{-1}\right.$ in PBS, ThermoFisher D1830) was injected directly in the eye of the embryo to visualize the blood vessels. The diameters of the blood vessels were measured over time with the software ImageJ.

For the experiment on calcium signaling, electrodes were placed on U87-GCamp6f spheroids one or two days after grafting. The voltage was in a range of $5-30 \mathrm{~V}$ corresponding to $1-6 \mathrm{kV} \mathrm{cm}^{-1}$. Fluorescence data extraction was made using the software ImageJ, respecting the following steps. First the spheroids were automatically detected using the threshold function of ImageJ and regions of interest data over time were exported into Excel. Values were then corrected by linear regression to remove effect of photobleaching, and finally were normalized according to Eq. (2):

$\Delta F=\frac{F(t)-F_{0}}{F_{0}}$

$F(t)$ corresponds to fluorescence intensity at a given time $t$ and $F_{0}$ the average fluorescence intensity calculated on the $10 \mathrm{~s}$ preceding the delivery of PEFs.

For both experiments, measures were done at least in triplicates, except for $30 \mathrm{~V}$ in the calcium signaling experiment, as there was most of the time appearance of bubbles disturbing the measurements. Negative controls were performed by placing the devices on the desired area but delivering no PEFs.

For the experiment on Propidium lodide (PI) uptake, $10 \mu \mathrm{L}$ of $15 \mu \mathrm{M} \mathrm{PI}$ were added on top of the vascularized spheroid and a picture of the initial fluorescence was taken, before placing the electrodes on top of the tumor. The voltage was set to $20 \mathrm{~V}$ and $1,4,10$, and 20 trains of 5 positive pulses of $100 \mu \mathrm{s}$ with a repetition frequency of $1 \mathrm{~Hz}$ were delivered every $30 \mathrm{~s}$. After removing the electrodes, $10 \mu \mathrm{L}$ of $\mathrm{PI}$ were added on top of the spheroid and a picture of the final fluorescence was taken, with the same parameters of exposure. A control was performed by following the protocol, but without delivering PEFs.

\section{Imaging by fluorescence microscopy}

Imaging was performed with a CMOS camera (Prime 95B, Photometrics) and an illumination system for fluorescence microscopy ( $p E-4000$, CoolLed). Images were recorded using the software WinFluor (University of Strathclyde, Glasgow). The blood vessels dyed with Dextran Texas Red 70,000 were visualized at $\lambda_{\text {exc }}=595 \mathrm{~nm}$. The U87 spheroids dyed with Calcein-AM and the U87-GCaMP6f spheroids were observed at $\lambda_{\text {exc }}=$ $490 \mathrm{~nm}$. The PI uptake was visualized at $\lambda_{\mathrm{exc}}=550 \mathrm{~nm}$. The values of fluorescence were corrected by using an exponential regression, in order to remove the effect of photobleaching of the dye.

\section{Two-photon imaging}

The preparation was positioned underneath a 16X objective (Nikon LWD $16 \mathrm{x} / 0.8 \mathrm{NA}$ ) under IR camera guidance, using $15 \mu \mathrm{M}$ PI in PBS as immersion fluid. Switching to two-photon mode, a control z-stack of the spheroid was acquired on a dual-scanhead two-photon microscope (FemtoS-Dual, Femtonics Ltd, Budapest, Hungary) equipped with a femtosecond pulsed laser tuned to $989 \mathrm{~nm}$ (Mai Tai HP, SpectraPhysics). Following the placement of the electrode, five $20 \mathrm{~V}$-pulses were applied with $100 \mu \mathrm{s}$ pulse width at $1 \mathrm{~Hz}$, repeated 10 times at $30 \mathrm{~s}$ interstimulus intervals. Then the electrode was removed and another $z$-stack with parameters identical to the control one was acquired. Data were analyzed using the MES software package (Femtonics Ltd., Budapest, Hungary) running under Matlab (Mathworks).

\section{DATA AVAILABILITY}

The data generated and analyzed during the study are available from the corresponding author.

Received: 29 March 2021; Accepted: 16 July 2021; Published online: 13 August 2021

\section{REFERENCES}

1. Tamimi, A. F. \& Juweid, M. Epidemiology and Outcome of Glioblastoma. in Glioblastoma (ed. De Vleeschouwer, S.) (Codon Publications, 2017).

2. Koshy, M. et al. Improved survival time trends for glioblastoma using the SEER 17 population-based registries. J. Neurooncol. 107, 207-212 (2012).

3. Wilson, T. A., Karajannis, M. A. \& Harter, D. H. Glioblastoma multiforme: state of the art and future therapeutics. Surg. Neurol. Int. 5, 64 (2014).

4. Davis, M. E. Glioblastoma: overview of disease and treatment. Clin. J. Oncol. Nurs. 20, S2-S8 (2016).

5. Lassman, A. B., Joanta-Gomez, A. E., Pan, P. C. \& Wick, W. Current usage of tumor treating fields for glioblastoma. Neuro-Oncol. Adv. 2, vdaa069 (2020).

6. Breton, M. \& Mir, L. M. Microsecond and nanosecond electric pulses in cancer treatments. Bioelectromagnetics 33, 106-123 (2012).

7. Gothelf, A., Mir, L. \& Gehl, J. Electrochemotherapy: results of cancer treatment using enhanced delivery of bleomycin by electroporation. Cancer Treat. Rev. 29, 371-387 (2003).

8. Gibot, L., Wasungu, L., Teissié, J. \& Rols, M.-P. Antitumor drug delivery in multicellular spheroids by electropermeabilization. J. Controlled Release 167, 138-147 (2013).

9. Dermol-Černe, J. et al. Plasma membrane depolarization and permeabilization due to electric pulses in cell lines of different excitability. Bioelectrochemistry 122, 103-114 (2018).

10. Frandsen, S. K. et al. Direct Therapeutic Applications of Calcium Electroporation to Effectively Induce Tumor Necrosis. Cancer Res. 72, 1336-1341 (2012).

11. Lee, E. W. et al. Advanced hepatic ablation technique for creating complete cell death: irreversible electroporation. Radiology 255, 426-433 (2010).

12. Edd, J. F., Horowitz, L., Davalos, R. V., Mir, L. M. \& Rubinsky, B. In vivo results of a new focal tissue ablation technique: irreversible electroporation. IEEE Trans. Biomed. Eng. 53, 1409-1415 (2006).

13. Brinton, M., Mandel, Y., Schachar, I. \& Palanker, D. Mechanisms of electrical vasoconstriction. J. NeuroEngineering Rehabil. 15, 43 (2018).

14. Sersa, G. et al. Vascular disrupting action of electroporation and electrochemotherapy with bleomycin in murine sarcoma. Br. J. Cancer 98, 388-398 (2008).

15. Bardet, S. M. et al. Multiphoton imaging reveals that nanosecond pulsed electric fields collapse tumor and normal vascular perfusion in human glioblastoma xenografts. Sci. Rep. 6, 34443 (2016).

16. Sersa, G. \& Cemazar, M. Vascular-disrupting action of electrochemotherapy: mode of action and therapeutic implications. in Clinical Aspects of Electroporation eds. Kee, S. T., Gehl, J. \& Lee, E. W., 83-91 (Springer New York, 2011).

17. Lee, J. H., Kim, H., Kim, J. H. \& Lee, S.-H. Soft implantable microelectrodes for future medicine: prosthetics, neural signal recording and neuromodulation. Lab. Chip 16, 959-976 (2016).

18. Lee, H., Bellamkonda, R. V., Sun, W. \& Levenston, M. E. Biomechanical analysis of silicon microelectrode-induced strain in the brain. J. Neural Eng. 2, 81-89 (2005).

19. Wei, Z. et al. A pliable electroporation patch (ep-Patch) for efficient delivery of nucleic acid molecules into animal tissues with irregular surface shapes. Sci. Rep. 5, 1-9 (2015).

20. Fattahi, P., Yang, G., Kim, G. \& Abidian, M. R. A review of organic and inorganic biomaterials for neural interfaces. Adv. Mater. 26, 1846-1885 (2014).

21. Someya, T., Bao, Z. \& Malliaras, G. G. The rise of plastic bioelectronics. Nature 540, 379-385 (2016).

22. Pampaloni, F., Reynaud, E. G. \& Stelzer, E. H. K. The third dimension bridges the gap between cell culture and live tissue. Nat. Rev. Mol. Cell Biol. 8, 839-845 (2007).

23. Kunz-Schughart, L. A., Freyer, J. P., Hofstaedter, F. \& Ebner, R. The use of 3-D cultures for high-throughput screening: the multicellular spheroid model. J. Biomol. Screen. 9, 273-285 (2004).

24. Friedrich, J., Seidel, C., Ebner, R. \& Kunz-Schughart, L. A. Spheroid-based drug screen: considerations and practical approach. Nat. Protoc. 4, 309-324 (2009). 
25. Carlsson, J. \& Acker, H. Relations between ph, oxygen partial pressure and growth in cultured cell spheroids. Int. J. Cancer 42, 715-720 (1988).

26. Ribatti, D. Chapter 5 chick embryo chorioallantoic membrane as a useful tool to study angiogenesis. Int. Rev. Cell Mol. Biol. 270 181-224 (Academic Press, 2008).

27. Valdes, T. I., Kreutzer, D. \& Moussy, F. The chick chorioallantoic membrane as a novel in vivo model for the testing of biomaterials. J. Biomed. Mater. Res. 62 273-282 (2002)

28. Krock, B. L., Skuli, N. \& Simon, M. C. Hypoxia-induced angiogenesis. Genes Cancer 2, 1117-1133 (2011).

29. Adair, T. H. \& Montani, J.-P. Overview of Angiogenesis. (Morgan \& Claypool Life Sciences, 2010).

30. Das, S. \& Marsden, P. A. Angiogenesis in glioblastoma. N. Engl. J. Med. 369, 1561-1563 (2013).

31. De Magalhães, N., Liaw, L.-H. L. \& Berns, M. An instruction on the in vivo shell-less chorioallantoic membrane 3-dimensional tumor spheroid model. Cytotechnology 62, 279-283 (2010)

32. Lecomte, A., Degache, A., Descamps, E., Dahan, L. \& Bergaud, C. In vitro and in vivo biostability assessment of chronically-implanted Parylene $C$ neural sensors. Sens. Actuators B Chem. 251, 1001-1008 (2017).

33. Selvakumaran, J., Hughes, M. P., Keddie, J. L. \& Ewins, D. J. Assessing biocompatibility of materials for implantable microelectrodes using cytotoxicity and protein adsorption studies. in 2nd Annual International IEEE-EMBS Special Topic Conference on Microtechnologies in Medicine and Biology. Proceedings (Cat. No. 02EX578) 261-264 (2002). https://doi.org/10.1109/MMB.2002.1002326.

34. Lecomte, A., Descamps, E. \& Bergaud, C. A review on mechanical considerations for chronically-implanted neural probes. J. Neural Eng. 15, 031001 (2018).

35. Law, K.-Y. Definitions for hydrophilicity, hydrophobicity, and superhydrophobicity: getting the basics right. J. Phys. Chem. Lett. 5, 686-688 (2014).

36. Mercanzini, A., Colin, P., Bensadoun, J.-C., Bertsch, A. \& Renaud, P. In vivo electrical impedance spectroscopy of tissue reaction to microelectrode arrays. IEEE Trans. Biomed. Eng. 56, 1909-1918 (2009).

37. Srinivasan, B. et al. TEER measurement techniques for in vitro barrier model systems. J. Lab. Autom. 20, 107-126 (2015).

38. Riffle, S. \& Hegde, R. S. Modeling tumor cell adaptations to hypoxia in multicellular tumor spheroids. J. Exp. Clin. Cancer Res. 36, 102 (2017).

39. Nishida, N., Yano, H., Nishida, T., Kamura, T. \& Kojiro, M. Angiogenesis in cancer. Vasc. Health Risk Manag 2, 213-219 (2006).

40. Gehl, J., Skovsgaard, T. \& Mir, L. M. Vascular reactions to in vivo electroporation: characterization and consequences for drug and gene delivery. Biochim. Biophys. Acta Gen. Subj. 1569, 51-58 (2002).

41. Batista Napotnik, T. \& Miklavčič, D. In vitro electroporation detection methods: an overview. Bioelectrochemistry 120, 166-182 (2018).

42. Palanker, D., Vankov, A., Freyvert, Y. \& Huie, P. Pulsed electrical stimulation for control of vasculature: temporary vasoconstriction and permanent thrombosis. Bioelectromagnetics 29, 100-107 (2008).

43. Sutera, S. P. \& Skalak, R. The History of Poiseuille's Law. Annu. Rev. Fluid Mech. 25 1-20 (1993).

44. Novickij, V., Tabasnikov, A., Smith, S., Grainys, A. \& Novickij, J. Analysis of planar circular interdigitated electrodes for electroporation. IETE Tech. Rev. 32, 196-203 (2015).

45. Berridge, M. J., Lipp, P. \& Bootman, M. D. The versatility and universality of calcium signalling. Nat. Rev. Mol. Cell Biol. 1, 11-21 (2000).

46. Jones, B. L. \& Smith, S. M. Calcium-sensing receptor: a key target for extracellular calcium signaling in neurons. Front. Physiol. 7, 116 (2016).

47. Hille, B. Ion Channels of Excitable Membranes. Ion Channels of Excitable Membranes 18, 814 (2001).

48. Dijk, G., Ruigrok, H. J. \& O'Connor, R. P. Influence of PEDOT:PSS coating thickness on the performance of stimulation electrodes. Adv. Mater. Interfaces 7, 2000675 (2020).

49. Proctor, C. M. et al. Electrophoretic drug delivery for seizure control. Sci. Adv. 4, eaau1291 (2018).
50. Waldherr, L. et al. Targeted chemotherapy of glioblastoma spheroids with an iontronic pump. Adv. Mater. Technol. 6, 2001302 (2021).

51. Chen, T.-W. et al. Ultra-sensitive fluorescent proteins for imaging neuronal activity. Nature 499, 295-300 (2013).

52. Walser, R. et al. Generation of co-culture spheroids as vascularisation units for bone tissue engineering. Eur. Cell. Mater. 26, 222-233 (2013).

53. Dijk, G., Rutz, A. L. \& Malliaras, G. G. Stability of PEDOT:PSS-coated gold electrodes in cell culture conditions. Adv. Mater. Technol. 5, 1900662 (2020).

\section{ACKNOWLEDGEMENTS}

The work reported here was supported by the French National Research Agency (ANR-18-CE19-0029). We would like to thanks Nathan Dumas, Dr Séverine De Mulatier and Lionel Fliegans for their contribution to the mechanical characterization of the flexible probes, and Denise O'Connor for her help with the in ovo model.

\section{AUTHOR CONTRIBUTIONS}

R.P.O. contributed in the conception of the idea and the design of the project. M.C.L. contributed in the design, and performed experiments and data analysis. G.D. contributed in the microfabrication of the electrodes and the impedance spectroscopy measurements. A.K. contributed in the performance and data analysis of the two-photon microscopy experiment. D.M. conducted numerical modeling. M.B. constructed the pulse generator and helped in the general experiments. All authors contributed to the writing and the review of the manuscript.

\section{COMPETING INTERESTS}

The authors declare no competing interests.

\section{ADDITIONAL INFORMATION}

Supplementary information The online version contains supplementary material available at https://doi.org/10.1038/s41528-021-00115-x.

Correspondence and requests for materials should be addressed to R.P.O.

Reprints and permission information is available at http://www.nature.com/ reprints

Publisher's note Springer Nature remains neutral with regard to jurisdictional claims in published maps and institutional affiliations.

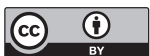

Open Access This article is licensed under a Creative Commons Attribution 4.0 International License, which permits use, sharing, adaptation, distribution and reproduction in any medium or format, as long as you give appropriate credit to the original author(s) and the source, provide a link to the Creative Commons license, and indicate if changes were made. The images or other third party material in this article are included in the article's Creative Commons license, unless indicated otherwise in a credit line to the material. If material is not included in the article's Creative Commons license and your intended use is not permitted by statutory regulation or exceeds the permitted use, you will need to obtain permission directly from the copyright holder. To view a copy of this license, visit http://creativecommons. org/licenses/by/4.0/.

(c) The Author(s) 2021 